\title{
Neurodegenerative networking
}

In neurodegenerative diseases, pathology does not spread like a fan from one brain area to neighbouring areas. Instead, the spread follows disease-specific patterns that resemble the architecture of brain connectivity networks. What remains unclear is why pathology spreads along such networks and whether the strength of network connectivity predicts the severity of neurodegeneration. Two new studies involving graph theoretical analysis of healthy brain connectivity suggest that in these disorders, neurodegenerative pathology spreads transneuronally through neural networks.

Together, the results of these studies provide support for the hypothesis that the spread of neurodegeneration across neural networks occurs via transneuronal propagation.

Seeley and colleagues used resting-state functional MRI data from healthy volunteers to determine the functional connectivity of brain regions that are known to undergo atrophy in five different neurodegenerative diseases. They then applied graph theoretical analysis to assess whether the connectivity profile of a region in healthy brains is indicative of regional vulnerability in diseased brains.

Regions with a functional intrinsic connectivity network map that closely matched the pattern of atrophy for a particular disease were termed 'epicentres', and regions that showed the strongest functional connectivity to an epicentre showed the greatest vulnerability to atrophy. According to the authors, their findings are compatible with a transneuronal model of pathology spread, which is in line with recent suggestions that proteins implicated in various neurodegenerative disorders (such as amyloid- $\beta$ and tau in Alzheimer's disease) may spread via prion-like mechanisms.

In the second paper, Raj et al. explicitly modelled the spread of prion-like neurodegenerative pathology by a network diffusive mechanism. They first generated structural connectivity networks from tractography data (obtained using structural MRI) from 14 healthy young individuals and then used graph theoretical analysis to model diffusive progression of pathology along the fibre pathways in these networks. This modelling revealed several spatially distinct patterns so-called 'eigenmodes' - by which such progression could proceed.

The most likely eigenmodes revealed by the model resembled patterns of atrophy in various dementias. Strikingly, the authors were able to model the persistence of eigenmodes as a function of age, and they found that the persistence of such modes with age correlated with the prevalence of the various dementias with age. The authors conclude that the classic patterns of neurodegenerative disease can be successfully reconstructed by a diffusive prion-like propagation model.
Together, the results of these studies provide support for the hypothesis that the spread of neurodegeneration across neural networks occurs via transneuronal propagation. The studies also demonstrate the power of graph theoretical analysis to develop and probe models of neural network breakdown in neurodegenerative disease.

Darran Yates

ORIGINAL RESEARCH PAPERS Zhou, J. et al. Predicting regional neurodegeneration from the healthy brain functional connectome. Neuron 73 , 1216-1227 (2012) | Raj, A., Kuceyeski, A. \& Weiner, M. A network diffusion model of disease progression in dementia. Neuron 73, 1204-1215 (2012)

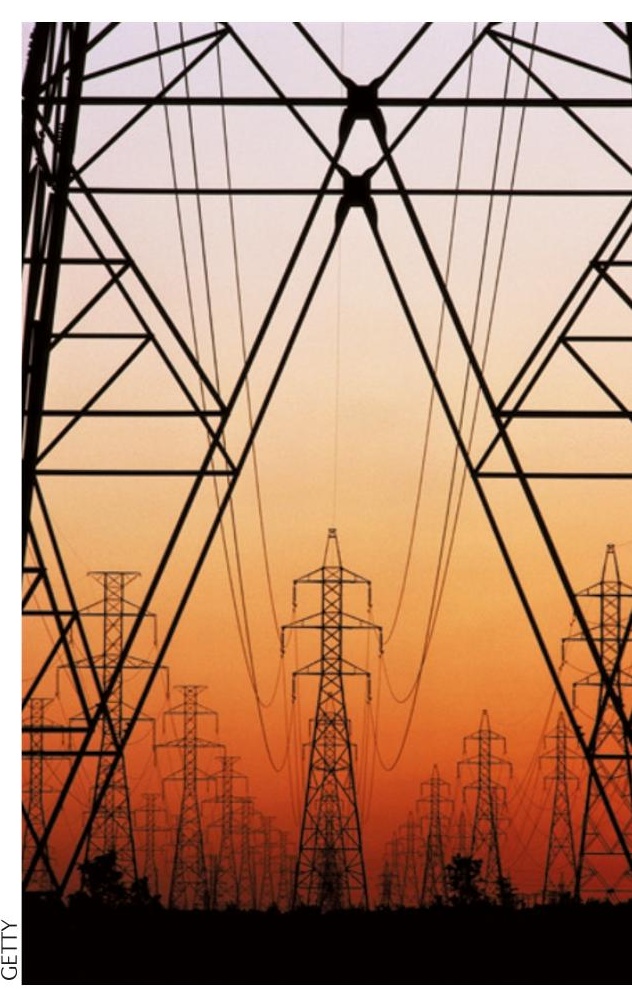

